\title{
The Intrinsic Beauty of Polytropic Spheres in Reduced Variables
}

\author{
Roberto Caimmi \\ Independent Researcher, Padova, Italy \\ Email: roberto.caimmi@unipd.it
}

Received 9 May 2016; accepted 8 August 2016; published 11 August 2016

Copyright (C) 2016 by author and Scientific Research Publishing Inc.

This work is licensed under the Creative Commons Attribution International License (CC BY). http://creativecommons.org/licenses/by/4.0/

\section{(c) (i) Open Access}

\section{Abstract}

The concept of reduced variables is revisited with regard to van der Waals' theory and an application is made to polytropic spheres, where the reduced radial coordinate is $w=r / R=\xi / \Xi, R$ radius, and the reduced density is $v=\rho / \lambda=\theta^{n}, \lambda$ central density. Reduced density profiles are plotted for several polytropic indexes within the range, $0 \leq n \leq 5$, disclosing two noticeable features. First, any point of coordinates, $(w, v), 0 \leq w \leq 1,0 \leq v \leq 1$, belongs to a reduced density profile of the kind considered. Second, sufficiently steep i.e. large $n$ reduced density profiles exhibit an oblique inflection point, where the threshold is found to be located at $n=n_{\text {th }}=\mathbf{0 . 8 8 8 7 1 5}$. Reduced pressure profiles, $q=p / \varpi=\theta^{n+1}, \varpi$ central pressure, Lane-Emden fucntions, $\theta=(\rho / \lambda)^{1 / n}$, and polytropic curves, $q=q(v)$, are also plotted. The method can be extended to nonspherical polytropes with regard to a selected direction, $w(\mu)=r(\mu) / R(\mu)=\xi(\mu) / \Xi(\mu)$. The results can be extended to polytropic spheres made of collisionless particles, for polytropic index within a more restricted range, $1 / 2 \leq n \leq 5$.

\section{Keywords}

Stars: Equilibrium, Galaxies: Equilibrium, Polytropic Spheres

\section{Introduction}

Models in reduced variables are useful tools for the description of the physical world, in that a single formulation relates to a whole set of configurations. For instance, let $\rho(r), 0 \leq r \leq R$, be the density profile of an as- 
signed mass distribution along a selected direction, and let $(r, \rho)=\left(r_{s}, \rho_{s}\right)$ be fixed nonzero scaling values. Let reduced (or scaled) variables be defined as $(w, v)=\left(r / r_{s}, \rho / \rho_{s}\right)$. Accordingly, the reduced density profile reads $v(w), 0 \leq w \leq W$, which includes an infinity of density profiles, $r \rightarrow k_{r} r, k_{r}>0, \rho \rightarrow k_{\rho} \rho, k_{\rho}>0$, in addition to the one under consideration.

A classical example of reduced variables can be found in van der Waals' theory of real gases in connection with the critical point, lying on the critical isothermal curve. The coordinates of the critical point on the Clapeyron plane are $P_{\mathrm{c}} \equiv\left(V_{\mathrm{c}}, p_{\mathrm{c}}, T_{\mathrm{c}}\right)$, where $V_{\mathrm{c}}$ is the largest volume along the critical isothermal curve still allowing a liquid phase, $p_{c}$ is the lowest pressure along the critical isothermal curve still allowing a liquid phase, and $T_{c}$ is the temperature along the critical isothermal curve i.e. the largest temperature still allowing a liquid phase.

Though isothermal curves on the Clapeyron plane, $(O V p)$, are different for different gases, the contrary holds on the reduced Clapeyron plane, $(\mathrm{OZq})$, where reduced isothermal curves coincide for all gases as $Z=V / V_{\mathrm{c}}$, $q=p / p_{\mathrm{c}}, U=T / T_{\mathrm{c}}$, with extension to ideal gases. In any case, the equation of state reads $p=p(V, T)$.

A special case of astrophysical interest, $p=p(V)$ or $p=p(\rho)$, relates to polytropic spheres or, in general, polytropes [1] Chap. IX, §§235-239 [2] [3], which are self-gravitating systems in hydrostatic equilibrium. Related scaling radius and scaling density are usually denoted as $r_{s}=\alpha$ and $\rho_{s}=\lambda$, respectively, where $\alpha$ depends on the central pressure, the central density, the density profile, and $\lambda$ is the central density.

In a widely investigated class of polytropic spheres, the reduced radial coordinate is denoted as $\xi=r / \alpha$ and the reduced density as $\theta^{n}=\rho / \lambda$, where $n$ is the polytropic index. The cases of astrophysical interest, $0 \leq n \leq 5$, range from null $(n=0)$ to infinite $(n=5)$ degree of concentration or, in other words, from homogeneous to Roche e.g., [1] Chap. IX, §§229-232 or Plummer [4] models, according if the central density is divergent or finite, respectively. The reduced radius, $\Xi=R / \alpha$, is a monotonically increasing function of the polytropic index, $n$, and $\Xi \rightarrow+\infty$ as $n \rightarrow 5$ [2] Chap. IV, §4 [3] Chap. 2, §2.5. Accordingly, the reduced density cannot be represented in a finite region of the reduced $\left(O \xi \theta^{n}\right)$ plane for $0 \leq n \leq 5$.

To this aim, a different choice of reduced radial coordinates has to be performed, $w=r / R=\xi / \Xi$, while the reduced density is left as $v=\rho / \lambda=\theta^{n}$. Under the restriction of null density on the boundary, $\theta^{n}=0$, the whole set of reduced density profiles on the $(O w v)$ plane lies within a square of unit sides parallel to the coordinate axes, with a vertex on the origin. A picture of the kind considered could be useful in disclosing additional features of reduced density profiles related to polytropic spheres. To this subject, the current investigation is devoted.

The paper is organized as follows. Reduced variables are introduced in Section 2, where the special case of ideal and real gases is presented as a guidance example. The special case of polytropic spheres is considered in Section 3, where reduced density profiles are plotted on the reduced $(O w v)$ plane for several values of the polytropic index, $0 \leq n \leq 5$, and the occurrence of an oblique inflection point is studied in detail. The reduced pressure profiles, the Lane-Emden functions, and the polytropic curves, are similarly considered therein. The discussion and the concluding remarks are drawn in Sections 4 and 5, respectively.

\section{Reduced Variables}

Let the equilibrium configuration of a thermodynamical system be defined by a set of physical parameters, $P_{1}, P_{2}, \cdots, P_{N}$. Let $P_{s, 1}, P_{s, 2}, \cdots, P_{s, N}$, be selected nonzero reference values or scaling parameters with respect to the above mentioned ones. Let the dimensionless parameters, $Q_{1}, Q_{2}, \cdots, Q_{N}$, be defined as reduced or scaled parameters, with respect to the above mentioned ones. A description in terms of reduced parameters includes all configurations where $P_{i} \rightarrow k_{i} P_{i}, k_{i}>0$; in particular, $k_{i}=1,1 \leq i \leq N$, relates to the system of interest.

As a guidance example, ideal and real gases shall be taken into consideration. For further details and exhaustive presentation, an interested reader is addressed to articles on the subject [5] [6] or specific textbooks [7] [8].

The equation of state of ideal [8] Chap. IV, §42 and real [9] gases, respectively, read:

$$
\begin{gathered}
p V=k N T ; \\
\left(p+A \frac{N^{2}}{V^{2}}\right)(V-N B)=k N T ;
\end{gathered}
$$

where $p$ is the pressure, $V$ the volume, $T$ the temperature, $N$ the particle number, $k$ the Boltzmann constant, $A$ and 
$B$ constants which depend on the nature of the particles. In particular, $B$ can be conceived as the volume filled by a particle of real gas and the product, $N B$, as the volume filled by all particles, or covolume [8] Chap. IV, $\S 74$.

Van der Waals' equation of state, Equation (2), can be rewritten as $p=p(V, T)$, and the partial derivatives, $(\partial p / \partial V)_{V, T},\left(\partial^{2} p / \partial V^{2}\right)_{V, T}$, can explicitly be expressed. Van der Waals isothermal curves may exhibit two extremum points (maximum and minimum), allowing a liquid phase, or no extremum point, allowing no liquid phase. The threshold relates to the critical isothermal curve, which shows a single extremum (horizontal inflection) point, where a liquid phase still occurs. The above mentioned inflection point is defined as critical point and related coordinates, $P_{\mathrm{c}} \equiv\left(V_{\mathrm{c}}, p_{\mathrm{c}}, T_{\mathrm{c}}\right)$, are defined as critical volume, critical pressure, critical temperature, respectively, where the last relates to the critical isothermal curve.

Owing to the mathematical properties of horizontal inflection points, $(\partial p / \partial V)_{V_{c}, T_{c}}=0,\left(\partial^{2} p / \partial V^{2}\right)_{V_{c}, T_{c}}=0$, which, together with $p_{\mathrm{c}}=p\left(V_{\mathrm{c}}, T_{\mathrm{c}}\right)$, make a system of three equations in the three unknowns, $V_{\mathrm{c}}, p_{\mathrm{c}}, T_{\mathrm{c}}, T_{\mathrm{c}}$. The solution is [7] Chap. XII, §20 [8] Chap. VIII, §85 [5] [6]:

$$
\begin{gathered}
V_{\mathrm{c}}=3 N B ; \\
T_{\mathrm{c}}=\frac{8}{27} \frac{A}{B} \frac{1}{k} ; \\
p_{\mathrm{c}}=\frac{1}{27} \frac{A}{B^{2}} ;
\end{gathered}
$$

in terms of the covolume, $N B$, and the constants, $A, B, k$.

With regard to the reduced variables:

$$
Z=\frac{V}{V_{\mathrm{c}}} ; \quad q=\frac{p}{p_{\mathrm{c}}} ; \quad U=\frac{T}{T_{\mathrm{c}}} ;
$$

the ideal gas equation of state, Equation (1), and van der Waals' equation of state, Equation (2), take the expression:

$$
\begin{gathered}
q Z=\frac{8}{3} U ; \\
\left(q+\frac{3}{Z^{2}}\right)\left(Z-\frac{1}{3}\right)=\frac{8}{3} U ;
\end{gathered}
$$

where the domain is $Z>0, Z>1 / 3$, respectively.

It is worth emphasyzing Equation (7), Equation (8) are independent of the nature of the gas, contrary to Equation (1), Equation (2), hence the great advantage of reduced variables with respect to physical variables. Reduced isothermal curves exhibit a horizontal asymptote, $q=0$, and a vertical asymptote, $Z=0$ and $Z=1 / 3$ for ideal and real gases, respectively. The reduced critical isothermal curve together a few neighbourhing ones, related to $U=20 / 23,20 / 22,20 / 21,20 / 20,20 / 19,20 / 18$, are shown in Figure 1 (full curves) together with their counterparts for ideal gases (dotted curves). The critical point is $P_{\mathrm{c}} \equiv(1,1,1)$.

\section{Polytropic Spheres}

\subsection{General Considerations}

Polytropes are special cases of barotropes i.e. self-gravitating fluids in hydrostatic equilibrium where the equation of state reads $p=p(\rho)$ or, restricting to polytropes [1] Chap. IX, §§235-239:

$$
p=K\left(\rho^{1+1 / n}-\rho_{\mathrm{b}}^{1+1 / n}\right) ;
$$

where $K$ is a constant, $n$ the polytropic index and $\rho_{\mathrm{b}}$ the density on the boundary, which is usually taken equal to zero [2] Chap. 4 [3] Chap. 2. The condition of hydrostatic equilibrium via Poisson equation reads [10]:

$$
\begin{gathered}
\Delta V_{\mathrm{G}}=-4 \pi G \rho ; \\
V_{\mathrm{G}}=K(n+1) \rho^{1 / n}+V_{\mathrm{b}} ;
\end{gathered}
$$




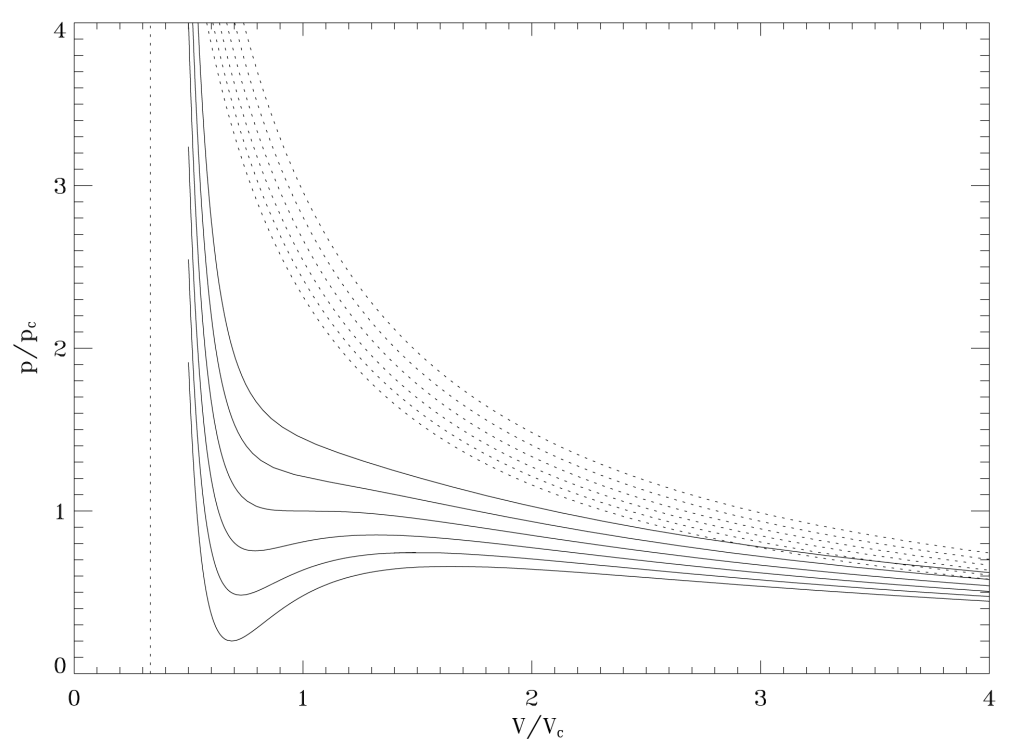

Figure 1. Isothermal curves in reduced variables for ideal (dotted) and van der Waals' (full) gases, respectively. The reduced temperature on each curve (from bottom to top in both cases) is $T / T_{c}=20 / 23,20 / 22,20 / 21,20 / 20,20 / 19,20 / 18$, respectively. The horizontal asymptote is the horizontal axis. The vertical asymptote of ideal isothermal curves is the vertical axis. The vertical asymptote of van der Waals' isothermal curves is shown as a dotted line, which defines the reduced covolume, $V / V_{c}=1 / 3$. No extremum point exists above the critical isothermal curve, $T / T_{c}=1$. See text for further details.

where $V_{\mathrm{G}}$ is the gravitational potential and $V_{\mathrm{b}}$ a normalization constant. From this point on, it shall be assumed $\rho_{\mathrm{b}}=0$ for simplicity.

Let $\lambda$ be the central density and $\alpha$ the scaling radius, defined as:

$$
\alpha=\left[\frac{(n+1) \varpi}{4 \pi G \lambda^{2}}\right]^{1 / 2}=\left[\frac{(n+1) K \lambda^{1 / n}}{4 \pi G \lambda}\right]^{1 / 2} ;
$$

where $\varpi$ is the central pressure and $K \lambda^{1+1 / n}$ is dimensioned as a pressure.

With regard to the reduced variables:

$$
\begin{gathered}
\xi=\frac{r}{\alpha} ; \quad 0 \leq r \leq R ; \\
v=\theta^{n}(\xi)=\frac{\rho(r)}{\lambda} ; \quad 0 \leq \xi \leq \Xi ;
\end{gathered}
$$

where $\Xi=R / \alpha$ is the reduced radius, the substitution of Equations (11)-(14) into (10) after some algebra yields the Lane-Emden equation [2] Chap. 4, §2 [3] [10] Chap. 2, §2.1:

$$
\theta^{\prime \prime}+\frac{2}{\xi} \theta^{\prime}=-\theta^{n}
$$

where the prime denotes derivation with respect to $\xi$.

Density profiles of astrophysical interest (i.e. decreasing with increasing radial coordinate) correspond to the range of polytropic index, $0 \leq n \leq 5$, where $n=0$ relates to homogeneous models and $n=5$ to Roche e.g., [1] Chap. IX, §§229-232 or Plummer [4] models, according if the central density, $\lambda$, is divergent or finite, respectively. Reduced radii are monotonically increasing from $\Xi=\sqrt{6}(n=0)$ to $\Xi \rightarrow+\infty(n=5)$.

The divergence of the reduced radius as $n \rightarrow 5$ makes a representation of reduced density profiles on the $(O \xi v)$ plane of little utility, in that interesting features could be lost. As $0 \leq \theta^{n} \leq 1$, a reduced radial coordinate within a similar range would be needed. To this respect, let an additional reduced radial coordinate be defined as: 


$$
w=\frac{r}{R}=\frac{\xi}{\Xi} ; \quad 0 \leq w \leq 1 ;
$$

and let the reduced density profile, $v=v(w)$, be considered and plotted on the (Owv) plane, which implies the knowledge of $\theta^{n}(\xi / \Xi), \Xi$, for selected values of $n$.

\subsection{Source of Data}

Physical parameters for sequences of rigidly rotating polytropes were determined in an earlier investigation [11], from nonrotating to maximally rotating (i.e. up to centrifugal support on the equatorial plane) configurations. Unfortunately, computations cannot be repeated as the original computer code is still in cards and no conversion into electronic format was tried in the past. For this reason, data used in the current paper are taken as specified below.

For polytropic indexes, $n=0,1,5$, where density profiles can be expressed analytically, standard formulae are used e.g., [10].

For integer and half-integer polytropic indexes, with the addition of $n=4.99$, seven-digit tables of LaneEmden functions [12] are used.

For quarter-integer polytropic indexes, with the addition of $n=0.001,0.010,0.050,0.100,0.200,0.808, a$ computer code with starting series solution followed by a fourth-order Runge-Kutta interpolation method, when an assigned tolerance is exceeded, is used.

Accordingly, reduced density profiles can be plotted on the (Owv) plane.

\subsection{Results}

Plotting in terms of the reduced radius, via Equation (16) implies the knowledge of the scaled radius, $\Xi$. Related values from the sources mentioned above are listed in Table 1 for several polytropic indexes, $n$, with regard to the computer code used in the present paper (pp), results from the parent paper [11] published later [13], and results from seven-digit tables of Lane-Emden functions [12]. The computer code does not hold for $n=0$ due to the occurrence of undetermined forms of the kind, $0 / 0$ and so on, and for $n=5$ due to memory overflow. An inspection of Table 1 discloses that, within $\theta(\Xi)<5 \times 10^{-7}, \Xi$ agrees with its counterpart from [11] and/ or [12] within a few percent for $0<n<0.5$ and within $10^{-4}$ or less for $0.75 \leq n \leq 4.75$.

Concerning reduced density profiles not included in the seven-digit tables of Lane-Emden functions [12], computed scaled radii, $\Xi$, are used in determining the reduced radial coordinate, $w=r / R=\xi / \Xi$, for the following reason. Within the range of interest, $0 \leq \xi \leq \Xi$, the Lane-Emden function, $\theta(\xi), 1 \geq \theta(\xi) \geq 0$, is monotonically decreasing, then overstimated/understimated $\Xi$ implies overstimated/understimated $\xi$ for fixed $\theta$, with respect to related true values listed in Table 1 , or in other words $\Delta \xi / \Delta \Xi>0$, where $\Delta \xi, \Delta \Xi$, are computation errors. Accordingly, the computed reduced radial coordinate reads $w=(\xi+\Delta \xi) /(\Xi+\Delta \Xi)$, which is closer to the true value, $w=\xi / \Xi$, than $w=(\xi+\Delta \xi) / \Xi$.

Reduced density profiles, $v=\rho(r) / \lambda=\theta^{n}(\xi)$, vs. reduced radial coordinates, $w=r / R=\xi / \Xi$, for polytropic index within the range, $0 \leq n \leq 5$, are plotted in Figure 2 where symbol captions are also listed in Table 1. Full $(n=0,1,5)$ and dashed $(n=0.1)$ curves with the addition of symbols $(n=0.25,0.808)$ are related to exact and computed (pp) solutions of the Lane-Emden equation, respectively. Remaining symbols (integer and half-integer $n, 1<n<5$, with the addition of $n=0.50,4.99$ ) are from seven-digit tables of Lane-Emden function [12].

The limiting case, $n=0$, is represented by the top and right side of the box in Figure 2 . To this respect, it is worth emphasyzing polytropic spheres with $n=0$ are conceptually different from MacLaurin spheres. More specifically, the latter are incompressible while the former are compressible but with infinite pressure inside and null pressure on the boundary conformly to hydrostatic equilibrium. Then the density on the boundary of polytropic spheres is null while it remains finite on the boundary of MacLaurin spheres, where a discontinuity arises.

The limiting case, $n=5$, is represented by the left and bottom side of the box in Figure 2, due to $\Xi \rightarrow+\infty$, which implies finite $\xi$ on the origin and infinite $\xi$ for the remaining of the domain, $0<\xi \leq \Xi$. In other words, the massive body is "compressed" into the origin while the vanishing atmosphere extends up to $\xi / \Xi=1$, similarly to Roche models in the physical space e.g., [1] Chap. IX, §§229-232.

Concerning the remaining cases, an inspection of Figure 2 shows reduced density profiles can be divided into 
Table 1. The scaled radius, $\Xi$, of polytropic spheres for polytropic index, $n$, within the range, $0 \leq n \leq 5$, according to the present paper (pp) and earlier investigations [11] [12]. Cases related to symbols (s) are plotted in Figure 2. See text for further details.

\begin{tabular}{|c|c|c|c|c|}
\hline$n$ & $\Xi$ (pp) & $\Xi[11]$ & $\Xi[12]$ & $\mathrm{s}$ \\
\hline 0.000 & & $2.44948974 \mathrm{E}+00$ & $2.44948974 \mathrm{E}+00$ & $\mathrm{f}$ \\
\hline 0.001 & $2.4499580 \mathrm{E}+00$ & & & \\
\hline 0.010 & $2.4547382 \mathrm{E}+00$ & $2.45488185 \mathrm{E}+00$ & & \\
\hline 0.050 & $2.4740123 \mathrm{E}+00$ & $2.47669981 \mathrm{E}+00$ & & \\
\hline 0.100 & $2.4977252 \mathrm{E}+00$ & $2.50454496 \mathrm{E}+00$ & & $\mathrm{~d}$ \\
\hline 0.200 & $2.5510944 \mathrm{E}+00$ & $2.56221918 \mathrm{E}+00$ & & \\
\hline 0.250 & $2.5797948 \mathrm{E}+00$ & $2.59208980 \mathrm{E}+00$ & & * \\
\hline 0.500 & $2.7404437 \mathrm{E}+00$ & $2.75269805 E+00$ & $2.75269805 \mathrm{E}+00$ & $\diamond$ \\
\hline 0.750 & $2.9345165 \mathrm{E}+00$ & $2.93451648 \mathrm{E}+00$ & & \\
\hline 0.808 & $2.9801387 \mathrm{E}+00$ & $2.98013932 \mathrm{E}+00$ & & + \\
\hline 1.000 & $3.1415925 \mathrm{E}+00$ & $3.14159265 E+00$ & $3.14159265 \mathrm{E}+00$ & $\mathrm{f}$ \\
\hline 1.250 & $3.3791024 \mathrm{E}+00$ & $3.37910200 \mathrm{E}+00$ & & \\
\hline 1.500 & $3.6537544 \mathrm{E}+00$ & $3.65375374 \mathrm{E}+00$ & $3.65375374 \mathrm{E}+00$ & $\Delta$ \\
\hline 1.750 & $3.9743875 \mathrm{E}+00$ & $3.97438776 \mathrm{E}+00$ & & \\
\hline 2.000 & $4.3528750 \mathrm{E}+00$ & $4.35287460 \mathrm{E}+00$ & $4.35287460 \mathrm{E}+00$ & $x$ \\
\hline 2.250 & $4.8055144 \mathrm{E}+00$ & $4.80551285 \mathrm{E}+00$ & & \\
\hline 2.500 & $5.3552750 \mathrm{E}+00$ & $5.35527546 \mathrm{E}+00$ & $5.35527546 \mathrm{E}+00$ & $\square$ \\
\hline 2.750 & $6.0355700 \mathrm{E}+00$ & $6.03557001 \mathrm{E}+00$ & & \\
\hline 3.000 & $6.8968500 \mathrm{E}+00$ & $6.89684862 \mathrm{E}+00$ & $6.89684862 \mathrm{E}+00$ & $*$ \\
\hline 3.250 & $8.0189350 \mathrm{E}+00$ & $8.01893753 \mathrm{E}+00$ & & \\
\hline 3.500 & $9.5358000 \mathrm{E}+00$ & $9.53580534 \mathrm{E}+00$ & $9.53580534 \mathrm{E}+00$ & $\diamond$ \\
\hline 3.750 & $1.1690285 \mathrm{E}+01$ & $1.16902937 \mathrm{E}+01$ & & \\
\hline 4.000 & $1.4971525 \mathrm{E}+01$ & $1.49715463 \mathrm{E}+01$ & $1.49715463 \mathrm{E}+01$ & + \\
\hline 4.250 & $2.0529055 \mathrm{E}+01$ & $2.05291013 \mathrm{E}+01$ & & \\
\hline 4.500 & $3.1836310 \mathrm{E}+01$ & $3.18364632 \mathrm{E}+01$ & $3.18364632 \mathrm{E}+01$ & $\Delta$ \\
\hline 4.750 & $6.6386250 \mathrm{E}+01$ & $6.63870957 \mathrm{E}+01$ & & \\
\hline 4.850 & $1.1295301 \mathrm{E}+02$ & & & $\mathrm{~d}$ \\
\hline 4.990 & & & $1.75818915 \mathrm{E}+03$ & $x$ \\
\hline 5.000 & & $+\infty$ & $+\infty$ & $\mathrm{f}$ \\
\hline
\end{tabular}

two main classes, namely exhibiting one or no oblique inflection point for $n \geq 1$ and $n \leq 0.808$, respectively, with the threshold lying in between. The monotonic trend of the reduced density, $v=\theta^{n}(\xi / \Xi)$, implies no extremum point and, in turn, oblique inflection points related to $\mathrm{d}^{2} v / \mathrm{d} w^{2}=0$, which via Equation (14) after little algebra can be expressed as:

$$
n \Xi^{2} \theta^{n-2}\left[(n-1)\left(\theta^{\prime}\right)^{2}+\theta \theta^{\prime \prime}\right]=0
$$




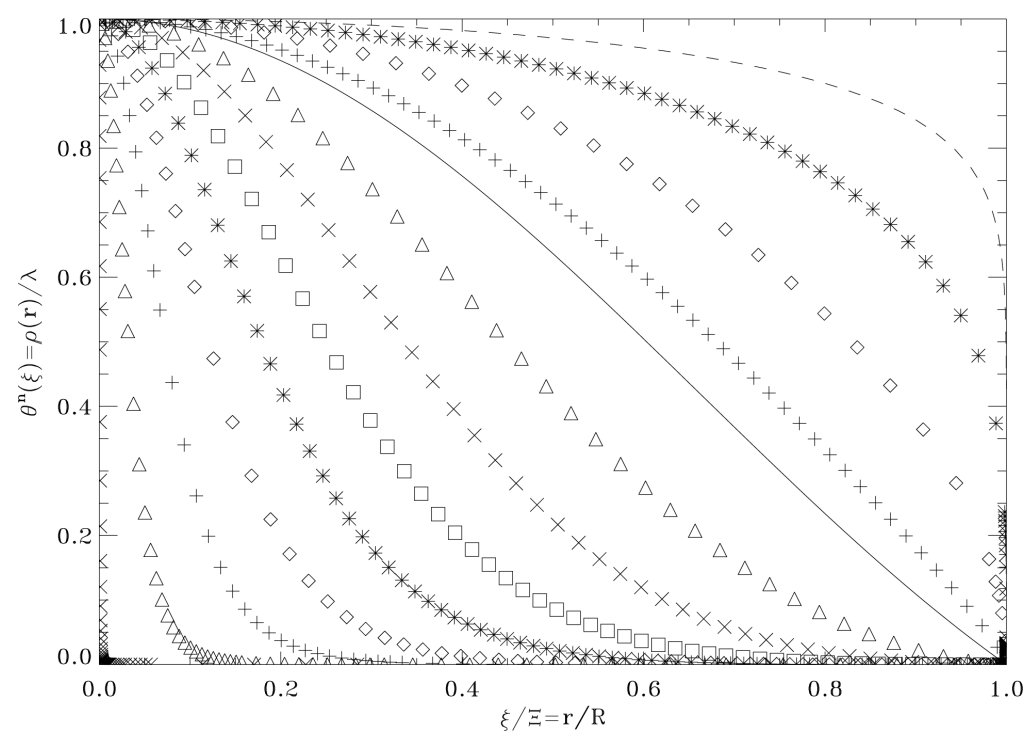

Figure 2. Density profiles of polytropic spheres in reduced variables, $\theta^{n}=\rho(r) / \lambda$ vs. $\xi / \Xi=r / R$, for different values of polytropic index, $0 \leq n \leq 5$, as listed in Table 1 , where the corresponding symbol is also shown. Full curves (f) relate to $n=0$ (top and right side of the box), $n=5$ (left and bottom side of the box), and $n=1$, for which density profiles can be expressed analytically. The dashed curve (d) relates to $n=0.1$. Symbols upside with respect to the full curve $(n=1)$ correspond to $n=0.25$ (asterisks), 0.5 (diamonds), 0.808 (crosses), starting from top right. Symbols downside with respect to the full curve correspond to $n=1.5$ (triangles), 2.0 (saltires), 2.5 (squares), 3.0 (asterisks), 3.5 (diamonds), 4.0 (crosses), 4.5 (triangles), 4.99 (saltires), starting from the full curve towards bottom left. Source of data: $n=0.5$ and $1<n<5$ [12]; $n=0.1,0.25,0.808$, (present paper). See text for further details.

where the prime denotes derivation with respect to $\xi$. The substitution of Equation (15) into (17) yields:

$$
(n-1)\left(\theta^{\prime}\right)^{2}-\frac{2}{\xi} \theta \theta^{\prime}-\theta^{n+1}=0
$$

which is the condition for the existence of an oblique inflection point in the case under discussion.

The lowest $n$ for which Equation (18) is still satisfied is found numerically using the computer code described above, and the result is $n=0.888715$ where $\Xi=3.0459945$ and the inflection point occurs at $\xi=2.650000$ within a tolerance $\varepsilon=5 \times 10^{-7}$. Related reduced density profile is not plotted in Figure 2 to avoid confusion.

Reduced pressure profiles, $q=p(r) / \varpi=\theta^{n+1}(\xi)$, vs. reduced radial coordinates, $w=r / R=\xi / \Xi$, for integer and half-integer polytropic index within the range, $0 \leq n \leq 5$, are plotted in Figure 3 where symbol captions are as in Figure 2 and data are from seven-digit tables of Lane-Emden function [12]. The limiting case, $n=5$, is represented by the left and bottom side of the box in Figure 3 .

An inspection of Figure 3 shows reduced pressure profiles can be divided into two main classes, namely exhibiting one or no oblique inflection point for $n \geq 0.5$ and $n<0.5$, respectively. The monotonic trend of the reduced pressure, $q=\theta^{n+1}(\xi / \Xi)$, implies no extremum point and, in turn, oblique inflection points related to $\mathrm{d}^{2} q / \mathrm{d} w^{2}=0$, which via Equation (14) after little algebra can be expressed as:

$$
(n+1) \Xi^{2} \theta^{n-1}\left[n\left(\theta^{\prime}\right)^{2}+\theta \theta^{\prime \prime}\right]=0
$$

where the prime denotes derivation with respect to $\xi$. The substitution of Equation (15) into (19) yields:

$$
n\left(\theta^{\prime}\right)^{2}-\frac{2}{\xi} \theta \theta^{\prime}-\theta^{n+1}=0
$$

which is the condition for the existence of an oblique inflection point in the case under discussion.

In the special case, $n=0$, the Lane-Emden function reads e.g., [10] $\theta(\xi)=1-\xi^{2} / 6$, hence $\theta^{\prime}(\xi)=-\xi / 3$ and Equation (20) reduces to: 


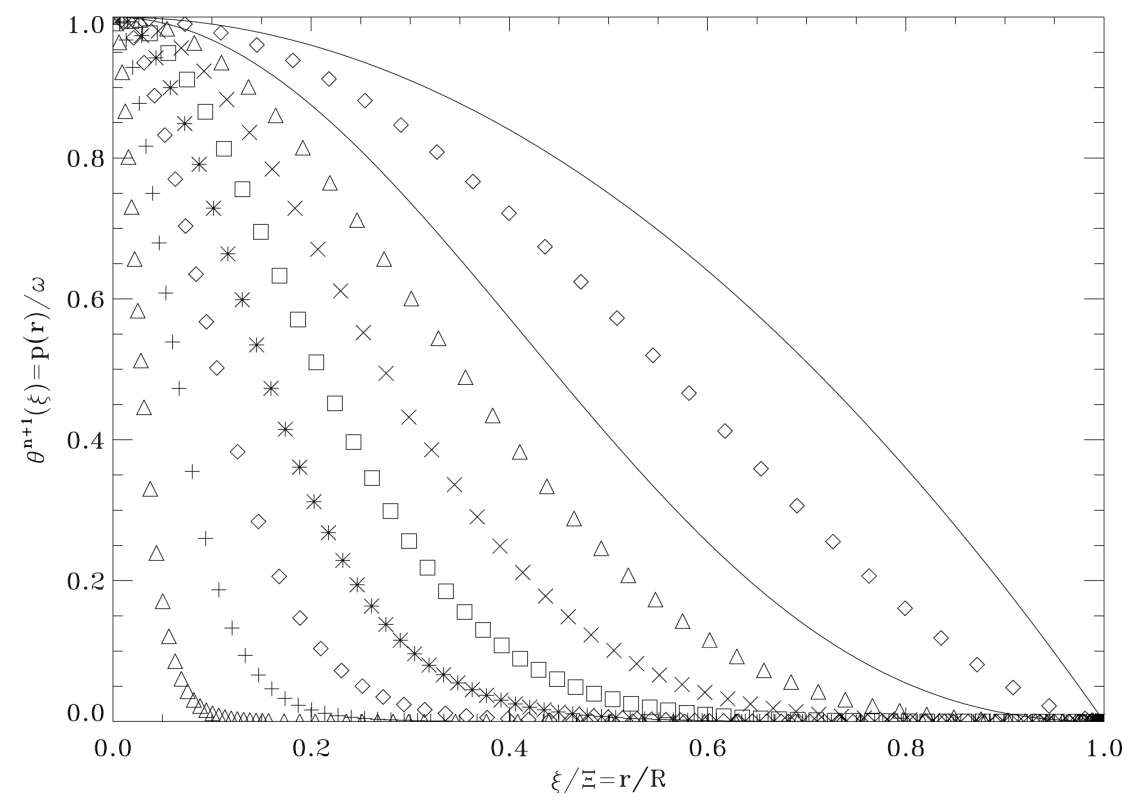

Figure 3. Pressure profiles of polytropic spheres in reduced variables, $\theta^{n+1}=p(r) / \varpi$ vs. $\xi / \Xi=r / R$, for integer and half-integer values of polytropic index, $0 \leq n \leq 5$, as listed in Table 1 , where the corresponding symbol is also shown. Full curves relate to $n=0$ (upper right), $n=5$ (left and bottom side of the box), and $n=1$, for which pressure profiles can be expressed analytically. Symbols correspond to $n=0.5$ (diamonds), 1.5 (triangles), 2.0 (saltires), 2.5 (squares), 3.0 (asterisks), 3.5 (diamonds), 4.0 (crosses), 4.5 (triangles), starting from the top right towards bottom left. Data are from seven-digit tables of Lane-Emden functions [12]. See text for further details.

$$
\theta^{n}=\frac{2}{3} ; \quad n=0
$$

which holds on the boundary, $\xi=\Xi=\sqrt{6}$, keeping in mind the pressure, and then the density, has to be null on the boundary. Then a "vertical" inflection point of the reduced pressure profile takes place on the boundary. Accordingly, all reduced pressure profiles, $q=p(r) / \varpi=\theta^{n+1}(\xi)$, within the range, $0 \leq n \leq 5$, exhibit an oblique inflection point.

The Lane-Emden functions, $\theta=[\rho(r) / \lambda]^{1 / n}$, vs. reduced radial coordinates, $w=r / R=\xi / \Xi$, for integer and half-integer polytropic index within the range, $0 \leq n \leq 5$, with the addition of $n=4.85,4.99$, are plotted in Figure 4 where symbol captions are as in Figure 2 and data are from seven-digit tables of Lane-Emden function [12] except for the case, $n=4.85$, where computations were performed as outlined above. The limiting case, $n=5$, is represented by the left and bottom side of the box in Figure 4 .

An inspection of Figure 4 shows the Lane-Emden function is characterized by the occurrence of an oblique inflection point, from the boundary $(n=0)$ to the centre $(n=5)$. In fact, the monotonic trend of the LaneEmden function, $\theta(\xi / \Xi)$, implies no extremum point and, in turn, oblique inflection points related to $\mathrm{d}^{2} \theta / \mathrm{d} w^{2}=0$, or $\Xi^{2} \theta^{\prime \prime}=0$, which via Equation (15) can be expressed as:

$$
\frac{2}{\xi} \theta^{\prime}=-\theta^{n}
$$

that in the special case, $n=0$, reduces to Equation (21). Then a "vertical" inflection point of the Lane-Emden function takes place on the boundary. Accordingly, all Lane-Emden functions, $\theta=[\rho(r) / \lambda]^{1 / n}$, within the range, $0 \leq n \leq 5$, exhibit an oblique inflection point.

The reduced polytropic curves, $q=q(v)$ or $\theta^{n+1}$ vs. $\theta^{n}$, for integer polytropic index within the range, $0 \leq n \leq 5$, with the addition of $n=0.50,0.25,0.10$, are plotted in Figure 5 where symbol captions are as in Figure 2 and data are from seven-digit tables of Lane-Emden function [12] except for the cases $n=0.25,0.10$, where computations were performed as outlined above. 


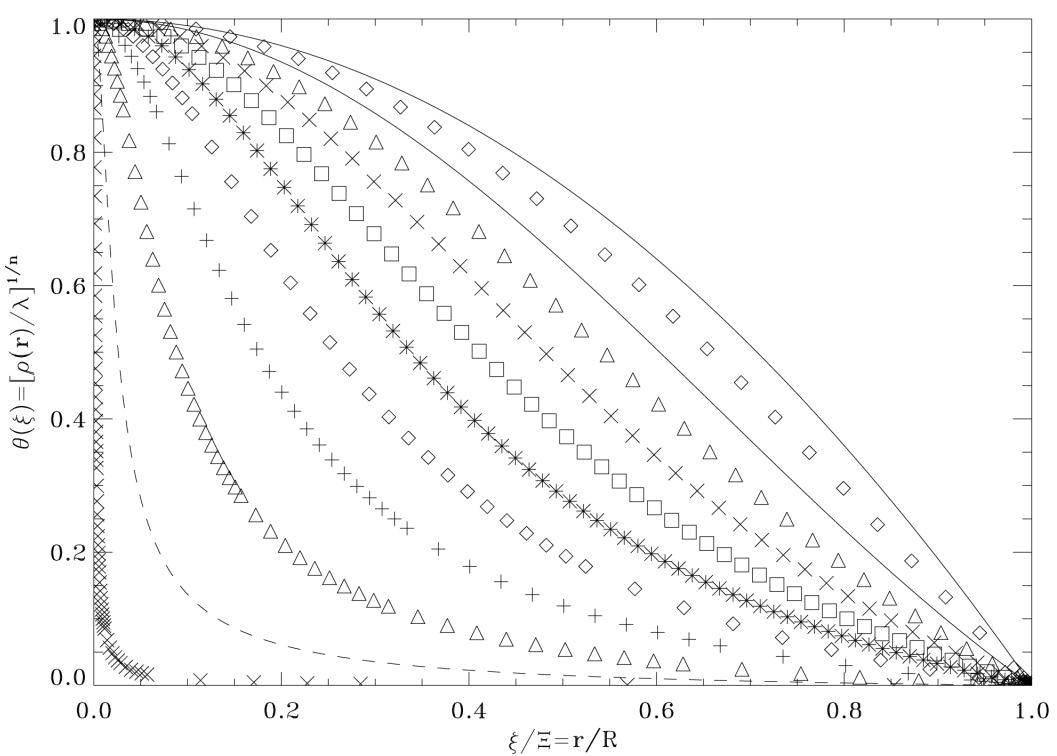

Figure 4. The Lane-Emden function in reduced variables, $\theta=[\rho(r) / \lambda]^{1 / n}$ vs. $\xi / \Xi=r / R$, for integer and half-integer values of polytropic index, $0 \leq n \leq 5$, with the addition of $n=4.85,4.99$, as listed in Table 1 , where the corresponding symbol is also shown. Full curves relate to $n=0$ (upper right), $n=5$ (left and bottom side of the box), and $n=1$, for which the Lane-Emden function can be expressed analytically. Symbols correspond to $n=0.5$ (diamonds), 1.5 (triangles), 2.0 (saltires), 2.5 (squares), 3.0 (asterisks), 3.5 (diamonds), 4.0 (crosses), 4.5 (triangles), 4.85 (dashed), 4.99 (saltires), starting from top right towards bottom left. Data are from seven-digit tables of Lane-Emden functions [12] except for $n=4.85$ (present paper). See text for further details.

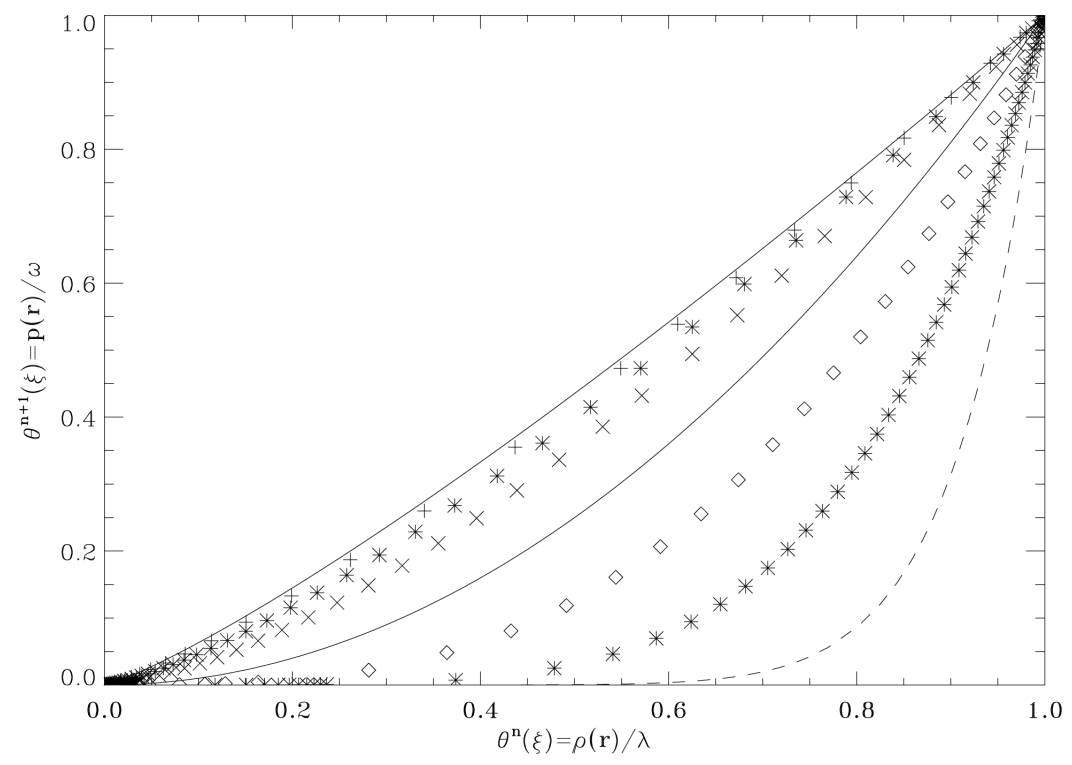

Figure 5. Reduced polytropic curves, $q=q(v)$ or $\theta^{n+1}$ vs. $\theta^{n}$, for integer values of polytropic index, $0 \leq n \leq 5$, with the addition of $n=0.50,0.25,0.10$, as listed in Table 1 , where the corresponding symbol is also shown. Full curves relate to $n=0$ (right and bottom side of the box), $n=5$ (upper left), and $n=1$, for which the Lane-Emden function can be expressed analytically. The dashed curve relates to $n=0.1$. Symbols correspond to $n=0.25$ (asterisks), 0.5 (diamonds), 2.0 (saltires), 3.0 (asterisks), 4.0 (crosses), starting from bottom right towards top left. Data are from seven-digit tables of Lane-Emden functions [12] except for $n=0.10,0.25$ (present paper). The centre and the boundary of the sphere correspond to $(1,1)$ and $(0,0)$, respectively. See text for further details. 
The centre and the boundary of the sphere correspond to $(1,1)$ and $(0,0)$, respectively. The limiting case, $n=0$, is represented by the right and bottom side of the box in Figure 5 .

\section{Discussion}

An application to polytropic spheres has shown the usefulness and the power of models in reduced variables. With regard to the plane, $(O w v)$, reduced density profiles for polytropic indexes, $0 \leq n \leq 5$, completely fill a square of unit side, $0 \leq w \leq 1,1 \geq v \geq 0$, where the top and the right side relate to homogeneous models $(n=0)$, while the left and the bottom side relate to Roche and Plummer models $(n=5)$. The last case can be related to extremely inhomogeneous mass distributions, where the extension is finite and the density is nonzero only on the centre for Roche models e.g., [1] Chap. IX, §§229-232, while the extension is infinite and the density is nonzero provided the distance from the centre remains finite for Plummer models [4].

Polytropic spheres can be conceived as matter distributions where the reduced slope, $\kappa=\mathrm{d} v / \mathrm{d} w$, lies between the extreme limits, $\kappa=0 \quad(n=0)$ and $\kappa \rightarrow-\infty \quad(n=5)$. From a geometrical point of view, the transition of reduced density profiles towards $\kappa \rightarrow 0$ or $n \rightarrow 0$ appears similar to the transition of Fermi-Dirac distribution functions towards zero absolute temperature, $T \rightarrow 0$ e.g., [8] Chap. V, §56.

The reduced slope, via Equation (14) and Equation (16) takes the explicit form:

$$
\kappa(\xi)=\frac{\mathrm{d} v}{\mathrm{~d} w}=n \Xi \theta^{n-1} \theta^{\prime}
$$

where $\theta^{\prime}$ remains finite. Accordingly, $\kappa(\Xi) \rightarrow-\infty$ for $0 \leq n<1, \kappa(\Xi)=1 / \pi$ for $n=1, \kappa(\Xi)=0$ for $1<n \leq 5$.

The occurrence of an oblique inflection point on reduced density profiles for sufficiently large $n$, with the threshold at $n=n_{\mathrm{th}}=0.888715$, suggests a definition of "steep" and "mild" reduced density profiles as related to $n \geq n_{\text {th }}$ and $n<n_{\text {th }}$, respectively, with regard to polytropic spheres. In addition, mild density profiles could be related to isothermal curves of real gases above the critical one, where no inflection point appears, and steep density profiles could be related to isothermal curves of real gases below the critical one, where (two extremum points and then) two inflection points appear, as shown in Figure 1.

The results of the current paper can be extended to polytropic spheres made of collisionless particles, keeping in mind collisionless polytropes in rigid rotation have an exact collisional counterpart within the range of polytropic index, $1 / 2 \leq n \leq 5$ [14].

The results can also be extended to nonspherical polytropes, provided reduced density profiles are considered along a selected direction, $r=r(\mu)$, hence $w(\mu)=r(\mu) / R(\mu)=\xi(\mu) / \Xi(\mu)$, while $v=\rho(r) / \lambda=\theta^{n}(\xi)$ remains unchanged in connection with isopycnic surfaces, $r=r(\mu)$ or $\xi=\xi(\mu), \mu=\cos \delta, \delta$ polar angle.

\section{Conclusion}

In conclusion, reduced variables appear to be not restricted to the Clapeyron plane as initially conceived [9], but they can be successfully extended to other physical situations as shown for polytropic spheres, which provides additional credit to van der Waals' original work.

\section{Acknowledgments}

The author is deeply indebted to G.P. Horedt for making available his FORTRAN program ${ }^{1}$ [12] and tables of Lane-Emden functions [3] in TEX format. Thanks are due to the Editor and the referee for their comments.

\section{References}

[1] Jeans, J. (1929) Astronomy and Cosmogony. Dover Publications, New York.

[2] Chandrasekhar, S. (1939) An Introduction to the Study of the Stellar Structure. University of Chicago Press, Chicago.

[3] Horedt, G.P. (2004) Polytropes: Applications in Astrophysics and Related Fields. Kluver Academic Publishers, Dordrecht.

\footnotetext{
${ }^{1}$ The program could not be used due to the lack of FORTRAN compiler in the author's computer. Calculations were performed using a GWBASIC program of lower but still acceptable precision.
} 
[4] Plummer, H.C. (1911) On the Problem of Distribution in Globular Star Clusters. Monthly Notices of the Royal Astronomical Society, 71, 460-470. http://dx.doi.org/10.1093/mnras/71.5.460

[5] Caimmi, R. (2010) A Principle of Corresponding States for Two-Component, Self-Gravitating Fluids. Serbian Astronomical Journal, 180, 19-55. http://dx.doi.org/10.2298/SAJ1080019C

[6] Caimmi, R. (2012) Tidal Interactions and Principle of Corresponding States: From Micro to Macro Cosmos. A Century after van der Waals’ Nobel Prize. http://arxiv.org/pdf/1210.3688.pdf

[7] Rostagni, A. (1957) Meccanica e Termodinamica. Ed. Libreria Universitaria di G. Randi, Padova.

[8] Landau, L.D. and Lifshitz, E.M. (1967) Physique Statistique. Mir, Moscow.

[9] van der Waals, J.D. (1873) Over de Continuited van den Gas-en Vloeistoftoestand. Doctoral Thesis, University of Leiden, Leiden.

[10] Caimmi, R. (1980) Emden-Chandrasekhar Axisymmetric, Solid-Body Rotating Polytropes. I-Exact Solutions for the Special Cases N Equals 0, 1 and 5. Astrophysics and Space Science, 71, 415-457.

[11] Caimmi, R. (1983) Emden-Chandrasekhar Axisymmetric, Solid-Body Rotating Polytropes. II-Power Series Solutions to EC Associated Equations of Degree 0 and 2. Astrophysics and Space Science, 89, 255-277. http://dx.doi.org/10.1007/BF00655979

[12] Horedt, G.P. (1986) Seven-Digit Tables of Lane-Emden Functions. Astrophysics and Space Science, 126, 357-408. http://dx.doi.org/10.1007/BF00639386

[13] Caimmi, R. (1985) Emden-Chandrasekhar Axisymmetric, Rigidly Rotating Polytropes. III—Determination of Equilibrium Configurations by an Improvement of Chandrasekhar's Method. Astrophysics and Space Science, 113, 125-142. http://dx.doi.org/10.1007/BF00650276

[14] Vandervoort, P.O. (1980) The Nonaxisymmetric Configurations of Uniformly Rotating Polytropes. The Astrophysical Journal, 241, 316-333. http://dx.doi.org/10.1086/158344

\section{Submit or recommend next manuscript to SCIRP and we will provide best service for you:}

Accepting pre-submission inquiries through Email, Facebook, LinkedIn, Twitter, etc.

A wide selection of journals (inclusive of 9 subjects, more than 200 journals)

Providing 24-hour high-quality service

User-friendly online submission system

Fair and swift peer-review system

Efficient typesetting and proofreading procedure

Display of the result of downloads and visits, as well as the number of cited articles

Maximum dissemination of your research work

Submit your manuscript at: http://papersubmission.scirp.org/ 